MODELING, IDENTIFICATION AND CONTROL, 1980, VOL. 1, NO. 1, 1-15

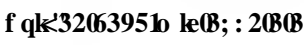

\title{
Estimation of synchronous machine parameters
}

\author{
ODDVAR HALLINGSTAD $\dagger$
}

Keywords: maximum likelihood method, identifiability, short circuit measurement, transient stability model, parameter estimation.

The present paper gives a short description of an interactive estimation program based on the maximum likelihood (ML) method. The program may also perform identifiability analysis by calculating sensitivity functions and the Hessian matrix. For the short circuit test the ML method is able to estimate the q-axis subtransient reactance $x^{\prime \prime}{ }_{\mathrm{q}}$, which is not possible by means of the conventional graphical method (another set of measurements has to be used). By means of the synchronization and close test, the ML program can estimate the inertial constant $(M)$, the d-axis transient open circuit time constant $\left(T^{\prime}{ }_{\mathrm{do}}\right)$, the d-axis subtransient o.c.t.c. $\left(T^{\prime \prime}{ }^{\circ}\right)$ and the q-axis subtransient o.c.t.c. $\left(T^{\prime \prime}{ }_{\mathrm{q}}\right)$. In particular, $T^{\prime \prime}{ }_{90}$ is difficult to estimate by any of the methods at present in use. Parameter identifiability is thoroughly examined both analytically and by numerical methods. Measurements from a small laboratory machine are used.

\section{Introduction}

The basic mathematical models of power systems, used in transient stability analysis, are deduced using physical laws and mathematical methods. In order to obtain simple models several assumptions and simplifications are made. There is then some uncertainty in the parameter values of the final models. The mathematical models may give a more accurate representation of the physical reality if the parameters are estimated (tuned) using measurements from the physical system.

\section{Short circuit test}

Short circuit tests are normally used to estimate many of the synchronous machine parameters. By using the estimation program described in the Appendix it is possible to determine the q-axis subtransient reactance, $x^{\prime \prime}{ }_{\mathrm{q}}$, in addition to the parameters normally estimated by the conventional graphical method.

\subsection{Experimental arrangement}

Several short circuit tests where made on a small laboratory machine at the Power System Laboratory of the Norwegian Institute of Technology. In these tests a d.c. machine was operated as a motor, driving the synchronous machine at rated speed. At time $t=0$, a switch was closed giving a symmetrical three-phase short circuit from no load. The armature current was recorded on a tape recorder, digitalized and read into a NORD-10 computer.

\subsection{Mathematical model}

This section presents a mathematical model of synchronous machines with an amortisseur winding subjected to a symmetrical three-phase short circuit from no

$\dagger$ The Norwegian Institute of Technology, Division of Engineering Cybernetics, N-7034 Trondheim. Norway. Present address: The Norwegian Defence Research Establishment, N-2007 Kjeller, Norway. 
load. The frequency is assumed to be constant at $50 \mathrm{~Hz}$ during the time that the terminals are shorted.

The short circuit armature current is deduced from the transient impedance matrix by means of Laplace analysis. The method can be found in Andersen (1976). The (phase a) short circuit current is given by

$$
\begin{aligned}
i(t)=U\left\{\left[\frac{1}{x_{\mathrm{d}}}+\left(\frac{1}{x_{\mathrm{d}}^{\prime}}-\frac{1}{x_{\mathrm{d}}}\right) \exp \left(-t / T_{\mathrm{d}}^{\prime}\right)+\left(\frac{1}{x_{\mathrm{d}}^{\prime \prime}}-\frac{1}{x_{\mathrm{d}}^{\prime}}\right) \exp \left(-t / T_{\mathrm{d}}^{\prime \prime}\right)\right]\right. \\
\times \cos (\omega t+\lambda)-\frac{1}{2}\left[\left(\frac{1}{x_{\mathrm{d}}^{\prime \prime}}+\frac{1}{x_{\mathrm{d}}^{\prime \prime}}\right) \cos \lambda+\left(\frac{1}{x_{\mathrm{d}}^{\prime \prime}}-\frac{1}{x_{\mathrm{d}}^{\prime \prime}}\right)\right. \\
\left.\times \cos (2 \omega t+\lambda)] \exp \left(-t / T_{\mathrm{a}}\right)\right\}
\end{aligned}
$$

where

$$
\begin{aligned}
x_{\mathrm{d}}: & \mathrm{d} \text {-axis synchronous reactance } \\
x^{\prime}{ }_{\mathrm{d}}: & \mathrm{d} \text {-axis transient reactance } \\
x^{\prime \prime}{ }_{\mathrm{d}}: & \mathrm{d} \text {-axis subtransient reactance } \\
x^{\prime \prime}{ }_{\mathrm{d}}: & \mathrm{q} \text {-axis subtransient reactance } \\
T_{\mathrm{d}}{ }_{\mathrm{d}}: & \mathrm{d} \text {-axis transient short circuit time constant } \\
U: & \text { open circuit terminal voltage (phase value) } \\
\omega: & \text { frequency of the armature current [rad/s] } \\
T^{\prime \prime}{ }_{\mathrm{d}}: & \text { d-axis subtransient short circuit time constant } \\
T_{\mathrm{a}}: & \text { armature winding time constant } \\
\lambda: & \text { phase angle at the instant when the terminals are shorted }
\end{aligned}
$$

The assumptions made in order to derive the armature current (1) from the transient impedance matrix are as follows:

(1) $r_{\mathrm{a}}{ }^{2}=0$, where $r_{\mathrm{a}}$ is the armature resistance.

(2) $\omega \gg 1 / T$ where $T$ denotes any of the time constants.

(3) $T_{\mathrm{d}}^{\prime} \gg T_{\mathrm{d}}^{\prime \prime}$.

In the conventional graphical method the unknown parameters are calculated using only the envelope of the armature current, drawn in a semilogarithmic diagram, see Andersen (1976). Since the q-axis subtransient reactance does not influence the peak values of the armature current, $i(t), x^{\prime \prime}{ }_{\mathrm{q}}$ is not identifiable by the conventional method (only the flanks of $i(t)$ are perturbed by changing $x^{\prime \prime}{ }_{q}$ ).

\subsection{Parameter estimation}

The mathematical model of the short circuit armature current (see eqn. (1)), is one solution form, and it has been derived assuming no process noise. A more realistic model would use a state space stochastic model instead of eqn. (1). However, the experiments confirmed that the modelling errors and process noise are so smalr that the deterministic description of the process dynamics is sufficient. The measurement is modelled by

$$
y\left(t_{k}\right)=i\left(t_{k}\right)+w\left(t_{k}\right)+b
$$

where $i\left(t_{k}\right)$ is given by eqn. (1) and the measurement noise consists of a bias term, $b$, and a white zero mean term, $w\left(t_{k}\right)$. The use of eqn. (1) has the advantage that no differential equations need to be solved in the estimation program. 
Since in this case zero process noise is assumed, the ML method is equal to the particular LS method called the output error method. Further $y\left(t_{k}\right)$ is a scalar measurement, its covariance is of no importance and we put it equal to one.

The eighth-order parameter vector

$$
p=\left[\lambda, \delta, T_{\mathrm{a}}, T_{\mathrm{d}}^{\prime}, T_{\mathrm{d}}^{\prime \prime}, x_{\mathrm{d}}^{\prime}, x_{\mathrm{d}}^{\prime \prime}, x_{\mathrm{q}}^{\prime \prime}\right]
$$

will be estimated.

The value of $x_{\mathrm{d}}$ is not estimated because it can be determined with sufficient accuracy from the steady-state measurements of $U$ and $i(t)$. The parameter estimates are shown in Table 1 for three cases.

(a) Parameter values estimated by the conventional graphical method.

(b) Parameter values estimated by the ML method, assuming $x^{\prime \prime}{ }_{\mathrm{q}} \neq x^{\prime \prime}{ }_{\mathrm{d}}$.

(c) Parameter values estimated by the ML method, assuming $x^{\prime \prime}{ }_{\mathrm{q}}=x^{\prime \prime}{ }_{\mathrm{d}}$ (giving seventh-order parameter vector).

\begin{tabular}{|c|c|c|c|}
\hline Parameter & $\begin{array}{l}\text { Graphical } \\
\text { method }\end{array}$ & $\underset{x^{\prime \prime}}{\text { ML method }} x_{{ }^{\prime \prime}}$ & $\begin{array}{c}\text { ML method } \\
x_{x_{\mathrm{q}}^{\prime \prime}}^{\prime \prime} x_{\mathrm{d}^{\prime \prime}}\end{array}$ \\
\hline$T_{\mathrm{a}}[\mathrm{s}]$ & 0.015 & 0.016 & 0.017 \\
\hline$T_{\mathrm{d}}^{\prime}[\mathrm{s}]$ & 0.052 & 0.057 & 0.015 \\
\hline$T^{\prime \prime}{ }_{\mathrm{d}}^{\prime \prime}[\mathrm{s}]$ & $0 \cdot 013$ & 0.012 & 0.054 \\
\hline$x_{\mathrm{d}}$ [p.u.] & $(1 \cdot 60)$ & $(1 \cdot 60)$ & $(1 \cdot 60)$ \\
\hline$x_{\mathrm{d}}^{\prime}[$ p.u.] & 0.37 & $0 \cdot 34$ & 0.26 \\
\hline$x_{\mathrm{d}}^{\prime \prime}$ [p.u.] & $0 \cdot 18$ & $0 \cdot 18$ & 0.17 \\
\hline$x^{\prime \prime}{ }_{\mathrm{q}}$ [p.u.] & & 0.41 & 0.17 \\
\hline
\end{tabular}

Table 1. Values for the short circuit parameter estimates $\left(x_{\mathrm{d}}\right.$ is estimated from steady-state measurements).

The measurement, $y(t)$, and the innovations $\left(\epsilon\left(x^{\prime \prime}{ }_{\mathrm{q}} \neq x_{{ }_{\mathrm{d}}}\right)\right.$ and $\left.\epsilon\left(x_{\mathrm{q}}^{\prime \prime}=x^{\prime \prime}{ }_{\mathrm{d}}\right)\right)$ for the two cases $(b)$ and $(c)$ are shown in Fig. 1. Figure 2 shows the power spectra for the innovations.

\subsection{Discussion}

Table 1 and Fig. 1 highlight the danger of using the ML method when it does not account for the second harmonic $\left(x^{\prime \prime}{ }_{\mathrm{q}}=x^{\prime \prime}{ }_{\mathrm{d}}\right)$, since it only minimizes

$$
J=\frac{1}{N} \sum_{i=1}^{N}\left(y_{i}-\bar{y}_{i}\right)^{2}
$$

The minimum value occurs when the estimation error, $\epsilon\left(x^{\prime \prime}{ }_{\mathrm{q}}=x^{\prime \prime}{ }_{\mathrm{d}}\right)$, is equally distributed on the flanks and the peak values (see Fig. 1). This leads to incorrect parameter values when it is assumed that $x_{\mathrm{q}}^{\prime \prime}=x^{\prime \prime}{ }_{\mathrm{d}}$ (we find $T_{{ }_{\mathrm{d}}}>T^{\prime}{ }_{\mathrm{d}}$ ). The power spectrum in Fig. 2 contains a considerable second-harmonic content indicating model error. 


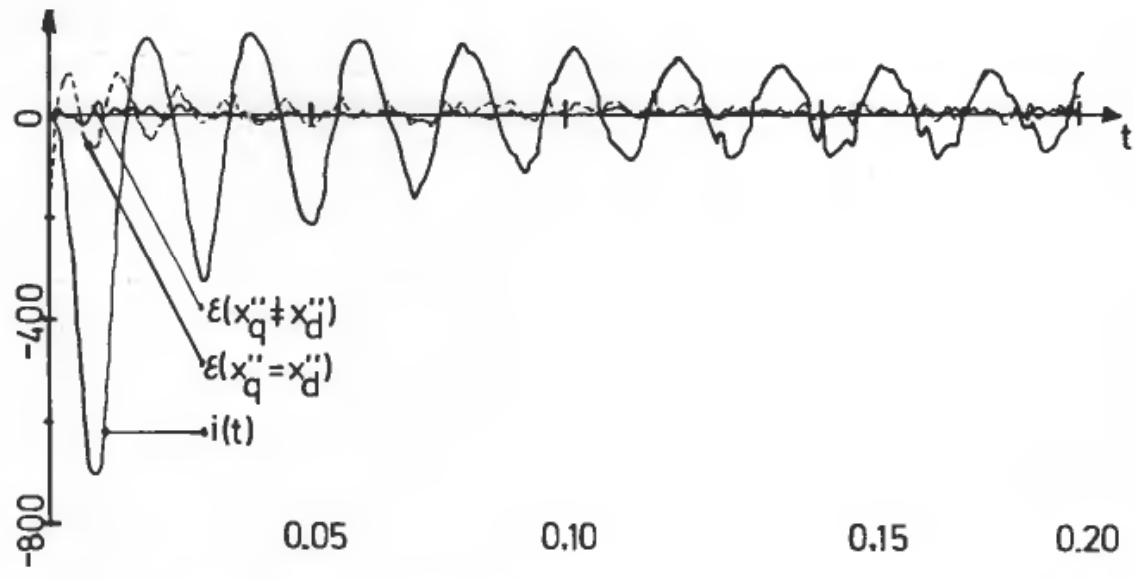

Figure 1. Measured armature current $(y)$ and innovations given by the ML method for the two cases $x^{\prime \prime}{ }_{\mathrm{q}} \neq x^{\prime \prime}{ }_{\mathrm{d}}$ and $x^{\prime \prime}{ }_{\mathrm{q}}=x^{\prime \prime}{ }_{\mathrm{d}}$.

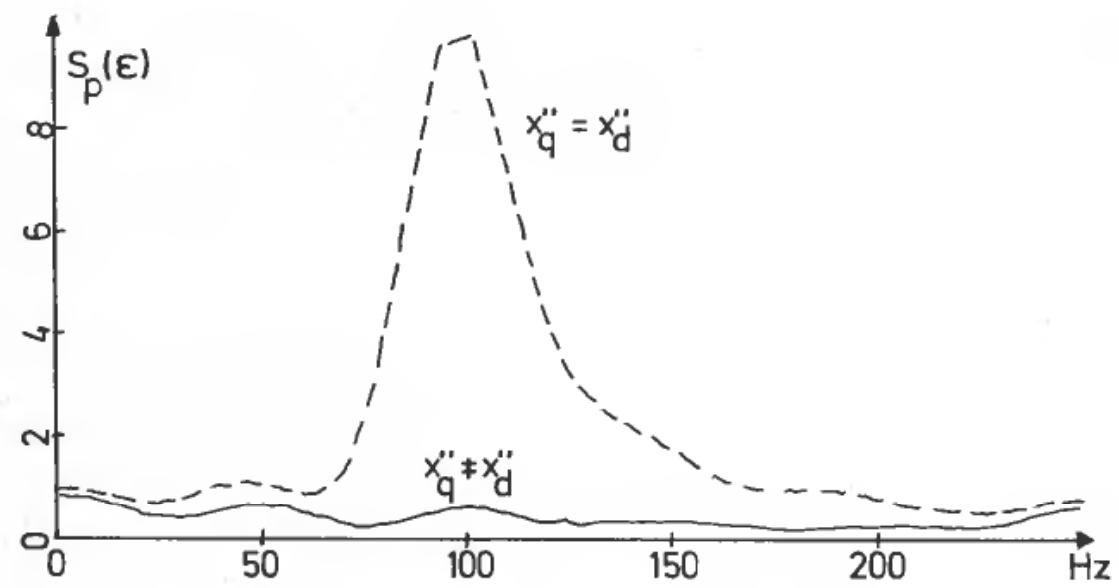

Figure 2. Power spectra for the innovations given by the ML method for the two cases $x^{\prime \prime}{ }_{\mathrm{q}} \neq x_{\mathrm{d}}^{\prime \prime}$ and $x_{\mathrm{q}}^{\prime \prime}=x^{\prime \prime}{ }_{\mathrm{d}}$.

Taking the second harmonic into account $\left(x^{\prime \prime}{ }_{\mathrm{q}} \neq x^{\prime \prime}{ }_{\mathrm{d}}\right)$ makes a great improvement as shown in Figs. 1 and 2 . The power spectrum has become almost flat indicating negligible model error. The agreement between the parameter values determined by the conventional method and the ML method using separate values for both $x^{\prime \prime}{ }_{\mathrm{q}}$ and $x_{\mathrm{d}}^{\prime \prime}$, is excellent (see Table 1).

Identifiability investigations by means of the Hessian matrix (see $\S$ A.2 of the Appendix) for the case where both $x^{\prime \prime}{ }_{\mathrm{q}}$ and $x^{\prime \prime}{ }_{\mathrm{d}}$ are estimated show that $T_{\mathrm{a}}$ and $T^{\prime}{ }_{\mathrm{d}}$ are more identifiable than $T^{\prime \prime}{ }_{\mathrm{d}}$. A $10 \%$ change in $T_{\mathrm{a}}$ or $T^{\prime}{ }_{\mathrm{d}}$ corresponds to a $20 \%$ change in $T^{\prime \prime}{ }_{\mathrm{d}}$. A similar investigation of the reactances shows that $x^{\prime \prime}{ }_{\mathrm{d}}$ is more identifiable than $x^{\prime}{ }_{\mathrm{d}}$ and $x^{\prime \prime}{ }_{\mathrm{q}}$, since changing $x^{\prime \prime}{ }_{\mathrm{d}}$ by 0.01 [p.u.] corresponds to change in $x_{d}^{\prime}$ of 0.03 [p.u.] or in $x_{q}^{\prime \prime}$ of 0.07 [p.u.]. 


\section{Synchronization and close test}

In this section the parameters of synchronous machine models used in transient stability programs are estimated. The most important parameters in this case are those which influence the electro-mechanical oscillatory mode. Some of these parameters cannot be estimated by the short circuit test described in the previous section (e.g. the q-axis open circuit subtransient time constant $T^{\prime \prime}{ }_{\text {qo }}$ and the moment of inertia $M$ ).

The identifiability investigation stands central to parameter estimation in dynamic modelling, because, as a rule, only some parameter aggregates are identifiable. Consequently identifiabilities are also investigated in this section.

\subsection{Experimental arrangement}

The same laboratory machine was used for these tests as in the short circuit tests of $\S 2.1$. The d.c. machine ran as a motor driving the synchronous machine at nominal speed with open terminals. The voltage difference $V_{\mathrm{D}}$ between the terminal of the synchronous machine and the corresponding network terminal (phase a) was measured as shown in Fig. 3.

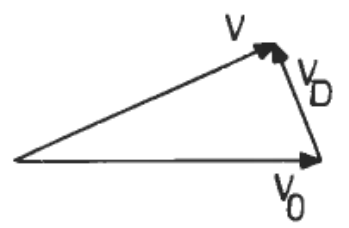

Figure 3. The phasor diagram before closing. $V$ : terminal voltage; $V_{0}$ : network voltage, $V_{\mathrm{D}}=V-V_{0}$

When $\left|V_{\mathrm{D}}\right|$ reached a predetermined value, a three-phase switch was closed, connecting the synchronous machine to the network. At the same time the d.c. machine was disconnected from its supply. The armature current of the synchronous machine was recorded on a tape recorder which was subsequently digitalized and read into the NORD-10 computer.

\subsection{Mathematical model}

At the network level the model is given by Fig. 4. Since it is assumed that $x^{\prime \prime}{ }_{q} \neq x^{\prime \prime}{ }_{\mathrm{d}}$ the e.m.f. $E_{\mathrm{G}}$ is current dependent.

At the machine level a model is chosen which represents the damper windings as two circuits, one in the direct axis and the other in the quadrature axis. In addition

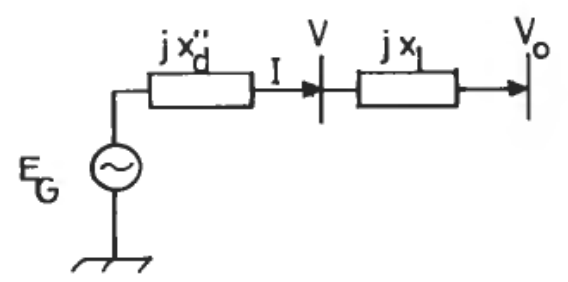

Figure 4. Description of the transient stability model at the network level. If $x^{\prime \prime}{ }_{\mathrm{q}} \neq x_{\mathrm{d}}{ }_{\mathrm{d}}$, the internal e.m.f., $E_{\mathbf{G}}$, is current dependent. 
we have one field circuit and two stator circuits. The differential equations describing the synchronous machine are as follows:

$$
\begin{gathered}
\dot{\beta}=\omega_{0}(\Omega-1) \\
\dot{\Omega}=\left(D_{\mathrm{tb}}-E^{\prime \prime}{ }_{\mathrm{d}} I_{\mathrm{d}}-E^{\prime \prime}{ }_{\mathrm{q}} I_{\mathrm{q}}-\left(x_{\mathrm{q}}^{\prime \prime}-x_{\mathrm{d}}^{\prime \prime}\right) I_{\mathrm{d}} I_{\mathrm{q}}\right) / M \\
\dot{E}^{\prime \prime}{ }_{\mathrm{d}}=\left(-E_{\mathrm{d}}^{\prime \prime}+\left(x_{\mathrm{q}}-x^{\prime \prime}{ }_{\mathrm{q}}\right) I_{\mathrm{q}}\right) / T^{\prime \prime}{ }_{\mathrm{q} \mathrm{o}} \\
\dot{E}_{\mathrm{q}}^{\prime \prime}=\left(-E^{\prime \prime}{ }_{\mathrm{q}}+E_{\mathrm{q}}^{\prime}-\left(x_{\mathrm{d}}^{\prime}-x_{{ }_{\mathrm{d}}}^{\prime \prime}\right) I_{\mathrm{d}}\right) / T^{\prime \prime}{ }_{\mathrm{do}} \\
\dot{E}_{\mathrm{q}}^{\prime}=\left(-E_{\mathrm{q}}^{\prime}{ }_{\mathrm{q}}+E_{\mathrm{q} f \mathrm{o}}-\left(x_{\mathrm{d}}-x_{\mathrm{d}}^{\prime}\right) I_{\mathrm{d}}\right) / T^{\prime}{ }_{\mathrm{do}}
\end{gathered}
$$

The simplicity of the network shown in Fig. 4 enables us to solve the network equations analytically even when $x^{\prime \prime}{ }_{\mathrm{q}} \neq x_{{ }_{\mathrm{d}}}$. The solution relative to the dq-axis is:

$$
\begin{aligned}
& I_{\mathrm{d}}=\left(E^{\prime \prime}{ }_{\mathrm{q}}-V_{0} \cos \beta\right) /\left(x^{\prime \prime}{ }_{\mathrm{d}}+x_{1}\right) \\
& I_{\mathrm{q}}=\left(V_{0} \sin \beta-E^{\prime \prime}{ }_{\mathrm{d}}\right) /\left(x^{\prime \prime}{ }_{\mathrm{q}}+x_{1}\right)
\end{aligned}
$$

The symbols used in eqns. (5)-(11) are as follows:

$\beta:$ angle between the rotor position and the synchronous reference

$\omega_{0}$ : prefault system angular velocity

$\Omega$ : per-unit rotor angular velocity

$D_{\mathrm{tb}}$ : constant friction torque

$E_{{ }_{d}}$ : d-axis component of voltage behind subtransient reactance

$E^{\prime \prime}{ }_{q}:$ q-axis component of voltage behind subtransient reactance

$E^{\prime}{ }_{\mathrm{q}}$ : field flux-linkages

$E_{\text {qfo }}$ : field voltage

$V_{0}$ : constant reference voltage

$I_{\mathrm{d}}:$ d-axis component of stator current

$I_{\mathrm{q}}$ : q-axis component of stator current

$M$ : inertia constant

$x_{\mathrm{d}}$ : d-axis synchronous reactance

$x_{d}^{\prime}$ : d-axis transient reactance

$x^{\prime \prime}{ }_{\mathrm{d}}: \mathrm{d}$-axis subtransient reactance

$x_{\mathrm{q}}$ : q-axis synchronous reactance

$x^{\prime \prime}{ }_{\mathrm{q}}: \mathrm{q}$-axis subtransient reactance

$x_{1}$ : line reactance

$T^{\prime}{ }_{\mathrm{do}}$ : d-axis transient open circuit time constant

$T^{\prime \prime}{ }_{\text {do }}$ : d-axis subtransient open circuit time constant

$T^{\prime \prime}{ }_{\text {qo }}$ : q-axis subtransient open circuit time constant

The mathematical model of eqns. (5)-(11) is non-linear because of the sine-cosine terms. Since no regulators were used in the tests no mathematical regulator models are given.

The relationship between the armature current $i(t)$ (phase a), which was measured, and the dq-axis current in eqns. (10) and (11) is given by the Park transformation,

$$
i(t)=I_{\mathrm{d}} \cos \left(\omega_{0} t+\beta+\lambda\right)-I_{\mathrm{q}} \sin \left(\omega_{0} t+\beta+\lambda\right)
$$

where $\lambda$ is the phase angle of $i(t)$.

Assuming a white measurement noise $w(t)$, our measurement equation becomes

$$
y(t)=i(t)+w(t)
$$




\subsection{Identifiability}

A direct investigation of the identifiability of the parameters in eqns. (5)-(13) is difficult because of the non-linearities. In order to perform an analytical identifiability investigation the following simplifying assumptions are made:

(1) Because of the uniqueness of the Park transformation it is permissible, in the absence of measurement noise, to use eqns. (10) and (11) as the measurement equations instead of eqn. (12); i.e. assuming $y_{1}=I_{\mathrm{q}}$ and $y_{2}=I_{\mathrm{d}}$.

(2) $D_{\mathrm{tb}}=0$ (friction torque).

(3) Equations (5)-(11) are linearized about the steady-state values:

$$
\left.\begin{array}{cc}
\beta_{0}=0 & E_{\mathrm{qo}}^{\prime \prime}=V_{0} \\
\Omega_{0}=1 \cdot 0 & E_{\mathrm{qo}}^{\prime}=V_{0} \\
E_{\mathrm{do}}^{\prime \prime}=0 & E_{\mathrm{qfo}}=V_{0}
\end{array}\right\}
$$

The assumptions above decouple the $\mathrm{d}-$ and $\mathrm{q}-\mathrm{axes}$, and lead to the following two characteristic equations:

$$
\begin{array}{r}
\ddot{y}_{1}+p_{2} \ddot{y}_{1}+p_{1} \dot{y}_{1}+p_{0} y_{1}=0 \\
\ddot{y}_{2}+q_{1} \dot{y}_{2}+q_{0} y_{2}=0
\end{array}
$$

where

$$
\begin{aligned}
& \left.\begin{array}{l}
p_{0}=\frac{V_{0}^{2} \omega_{0}}{\left(x_{\mathrm{q}}^{\prime \prime}+x_{1}\right) M} \cdot \frac{1}{T_{{ }_{\mathrm{q}}}^{\prime \prime}} \\
p_{1}=\frac{{V_{0}}^{2} \omega_{0}}{\left(x_{\mathrm{q}}^{\prime \prime}+x_{1}\right) M} \\
p_{2}=\frac{x_{1}+x_{\mathrm{q}}}{\left(x_{\mathrm{q}}^{\prime \prime}+x_{1}\right) T_{\mathrm{q}}^{\prime \prime}}
\end{array}\right\} \\
& \left.q_{0}=\frac{x_{1}+x_{\mathrm{d}}}{\left(x_{\mathrm{d}}^{\prime \prime}+x_{\mathrm{l}}\right) T^{\prime \prime}{ }_{\mathrm{do}}} \cdot \frac{1}{T_{\mathrm{do}}^{\prime}}\right) \\
& \left.q_{1}=\frac{x_{1}+x_{\mathrm{d}}^{\prime}}{\left(x_{\mathrm{d}}^{\prime \prime}+x_{1}\right) T_{\mathrm{do}}^{\prime \prime}}+\frac{1}{T_{\mathrm{do}}^{\prime}}\right\}
\end{aligned}
$$

Equations (15)-(18) tell us that only five parameter aggregates $p_{0}, p_{1}, p_{2}, q_{0}$ and $q_{1}$ may be identified in the linearized equations. These parameter aggregates may be estimated regardless of the state variables. $T^{\prime \prime}{ }_{\text {qo }}$ may always be estimated because

$$
T^{\prime \prime}{ }_{\mathrm{q} 0}=p_{1} / p_{0}
$$

Therefore, at most four parameters are identifiable in addition to $T^{\prime \prime}{ }_{\mathrm{q} 0}$, and they must yield a solution to eqns. (17) and (18) which is unique.

Even though there is an one-to-one correspondence between the parameters of the characteristics equation and the eigenvalues, the latter give more information about the degree of identifiability of parameters, because measurement of $y$ indicates which 
are the dominant eigenvalues. For instance, a poorly excited and very fast mode may drown in the measurement noise. When nominal values for the parameters are inserted in eqns. (17) and (18) the eigenvalues of eqns. (15) and (16) have the following approximate relationships to the synchronous machine parameters:

$$
\begin{aligned}
& \lambda_{1} \approx-\frac{1}{T^{m}{ }_{\mathrm{q}}} \frac{x_{1}+x_{\mathrm{q}}}{x_{1}+x^{\prime \prime}{ }_{\mathrm{q}}} \quad(=-205 \cdot 3) \\
& \operatorname{Re}\left\{\lambda_{2}\right\} \approx-\frac{V_{0}^{2} \omega_{0} T^{\prime \prime}{ }_{\mathrm{q}}}{2 M} \frac{x_{\mathrm{q}}-x_{\mathrm{q}}^{\prime \prime}}{\left(x_{1}+x_{\mathrm{q}}\right)^{2}} \quad(=-3.02)
\end{aligned}
$$

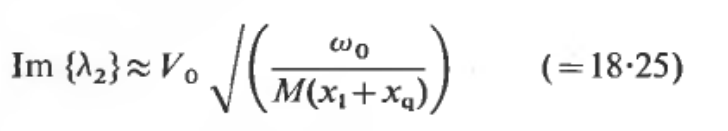

$$
\begin{aligned}
& \lambda_{4} \approx-\frac{1}{T_{\mathrm{do}}^{\prime}} \frac{x_{1}+x_{\mathrm{d}}}{x_{1}+x_{\mathrm{d}}^{\prime}} \quad(=-17 \cdot 43) \\
& \lambda_{5} \approx-\frac{1}{T^{\prime \prime}{ }_{\mathrm{do}}} \frac{x_{1}+x_{\mathrm{d}}^{\prime}}{x_{1}+x_{\mathrm{d}}^{\prime \prime}} \quad(=-82 \cdot 13)
\end{aligned}
$$

It is shown later that the dominating mode in the measurement is the oscillatory mode. Therefore $\operatorname{Re}\left\{\lambda_{2}\right\}$ and $\operatorname{Im}\left\{\lambda_{2}\right\}$ must have a high degree of identifiability. The first mode may be expected to be less identifiable because $\left|\lambda_{1}\right|$ is very large yet the measurement shows no sign of it. The degree of identifiability of $\lambda_{4}$ and $\lambda_{5}$ cannot be evaluated before the non-linear system is simulated (linearly they are not identifiable because they are not excited). There are therefore between two and four identifiable parameters.

\subsection{Parameter estimation}

Since it is found that the mathematical model is good and the process noise is negligible the ML method is equivalent to the LS method called the output error method. Because we have only one measurement its covariance value is of no importance and we can set it equal to one.

In the synchronization and close test, the system will not be in a steady state at the beginning of the estimation. This is because the armature current will have d.c.- and second-harmonic components when we connect the synchronous machine to the supply, with a phase difference between the two voltage vectors. The time $t=0$ for the synchronization and close test is therefore taken to be several periods after the closing time so that these components can decay. This is necessary because the d.c.second-harmonic components have been eliminated from the mathematical model given in eqns. (5)-(13). All the initial values of the state vector must therefore be estimated in addition to the phase angle $\lambda$, the initial field voltage $E_{\mathrm{qfo}}$, the constant friction torque $D_{\mathrm{tb}}$ and four synchronous machine parameters.

The unknown parameter vector of the synchronizing and close test is consequently

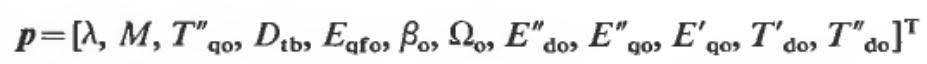

Only the four synchronous machine parameters $M, T^{\prime \prime}{ }_{\mathrm{qo}}, T^{\prime \prime}{ }_{\text {do }}$ and $T^{\prime \prime}{ }_{\text {do }}$ can be estimated because the fastest mode has a low degree of identifiability. 


\begin{tabular}{|c|c|c|c|c|c|c|}
\hline Case & $10^{4} \times \mathrm{J}_{\min }$ & $M$ & $T_{40}^{\prime \prime}$ & $T_{\mathrm{do}}^{\prime}$ & $T_{\mathrm{do}}^{\prime \prime}$ & $x^{\prime \prime}{ }_{\mathrm{q}}$ \\
\hline 1 & $3 \cdot 71$ & $1 \cdot 140$ & 0.0058 & 0.82 & & $x^{\prime \prime}{ }_{\mathrm{q}}=x_{\mathrm{d}}^{\prime \prime}$ \\
\hline 2 & $3 \cdot 48$ & $1 \cdot 136$ & $0 \cdot 0059$ & 0.51 & $0 \cdot 011$ & $x^{\prime \prime}{ }_{\mathrm{q}}=x_{\mathrm{d}}^{\prime \prime}$ \\
\hline 3 & $3 \cdot 68$ & $1 \cdot 144$ & $0 \cdot 0088$ & $0 \cdot 82$ & & \\
\hline 4 & $3 \cdot 47$ & $I \cdot 140$ & 0.0092 & 0.52 & 0.011 & \\
\hline \multicolumn{2}{|c|}{ Nominal value } & $1 \cdot 110$ & 0.0230 & $0 \cdot 27$ & 0.023 & 0.41 \\
\hline
\end{tabular}

Table 2. Results of the synchronization and close test. In cases of a missing entry the nominal value was used.

The results of the parameter estimation are presented in Table 2. The nominal values not given in Table 2 are

$$
\left.\begin{array}{ll}
x_{\mathrm{d}}=1 \cdot 60 \text { [p.u.] } & x_{\mathrm{q}}=0.85 \text { [p.u.] } \\
x_{\mathrm{d}}^{\prime}=0.34 \text { [p.u.] } & x_{1}=0.10 \text { [p.u.] } \\
x_{\mathrm{d}}^{\prime \prime}=0 \cdot 18 \text { [p.u.] } & V_{0}=1.00 \text { [p.u.] }
\end{array}\right\}
$$

\subsection{Discussion}

In this subsection Figs. 5-8 are commented after which several aspects of the estimation results presented in Table 2 and the figures are discussed.

The envelopes of the predicted value of $y$ agree excellently with the measured value as shown in Fig. 5. Figure 6 shows the power spectra of the innovations for all the cases in Table 2 . We see that all power spectra have a fifth harmonic component which is independent of the number of estimated parameters. This indicates an error in the model. The discrepancy is probably caused by non-sinusoidal distribution of the magnetic flux in the synchronous machine. When more parameters are used for the estimation, the power in the first harmonic is reduced. However, the reduction when estimating $T^{\prime \prime}{ }_{\text {do }}$, instead of using the nominal value, in addition to $M, T_{{ }_{\text {qo }}}^{\prime \prime}$ and $T^{\prime}{ }_{\text {do }}$ is negligible.

The parameters have been estimated with and without the assumption of subtransient symmetry $\left(x^{\prime \prime}{ }_{\mathrm{q}}=x^{\prime \prime}{ }_{\mathrm{d}}\right)$. It appears that only $T^{\prime \prime}{ }_{\mathrm{qo}}$ seems to depend strongly on

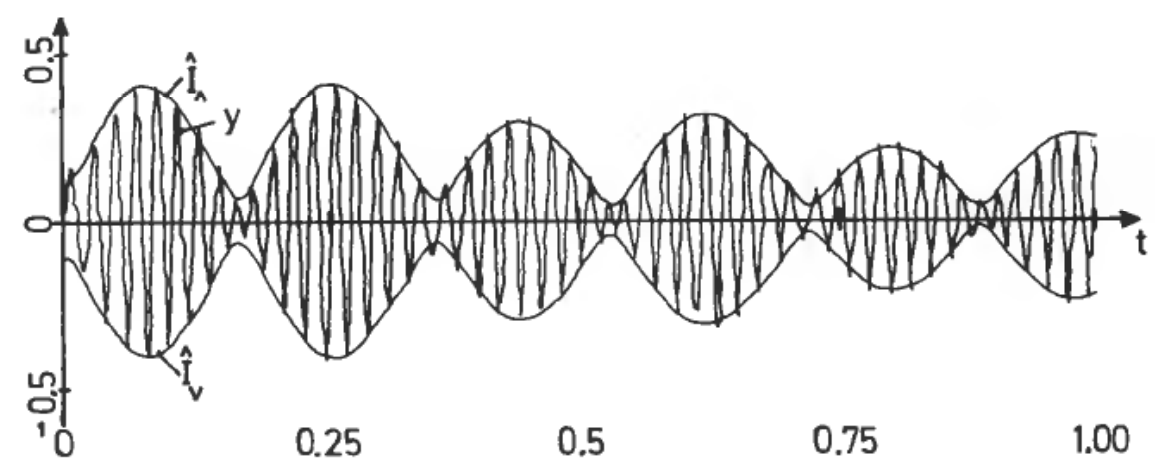

Figure 5. Measurement, $y$, and envelopes of the predicted measurement, $\hat{I}_{A}$ and $\hat{I}_{v}$, for Case 2 in Table 2. 
the value of $x^{\prime \prime}$. The reason for this dependency can be explained by eqn. (20). We find that $x^{\prime \prime}{ }_{9}$ influences the damping of the oscillatory mode, $\operatorname{Re}\left\{\lambda_{2}\right\}$. Because the damping has a high degree of identifiability, all estimates must give approximately the same value for $\operatorname{Re}\left\{\lambda_{2}\right\}$. Because the moment of inertia may be determined from the frequency term $\operatorname{Im}\left\{\lambda_{2}\right\}, T^{\prime \prime}{ }_{q 0}$ must satisfy

$$
T^{\prime \prime}{ }_{\mathrm{q} 0}\left(x_{\mathrm{q}}-x_{\mathrm{q}_{\mathrm{q}}}\right)=\text { constant }
$$

This implies that $T^{\prime \prime}{ }_{\mathrm{q} 0}$ must be a factor 1.5 greater when $x^{\prime \prime}{ }_{\mathrm{q}}=0.41$ than when $x^{\prime \prime}{ }_{\mathrm{q}}=x^{\prime \prime}{ }_{\mathrm{d}}=0.018$. This is consistent with the results in Table 2 . The conclusion is that if $T^{\prime \prime}{ }_{\text {qo }}$ is estimated rather than using the nominal value we may assume subtransient symmetry because this gives a model with the correct damping.

The analytical investigation of identifiability in $\S 3.3$ leads to the conclusion that the oscillatory mode has a high degree of identifiability. This is confirmed by Table 2, the parameter groups forming $\operatorname{Re}\left\{\lambda_{2}\right\}$ and $\operatorname{Im}\left\{\lambda_{2}\right\}$ (see eqn. (20)) remain constant in all the estimation cases. The two least identifiable eigenvalues are obviously $\lambda_{1}$
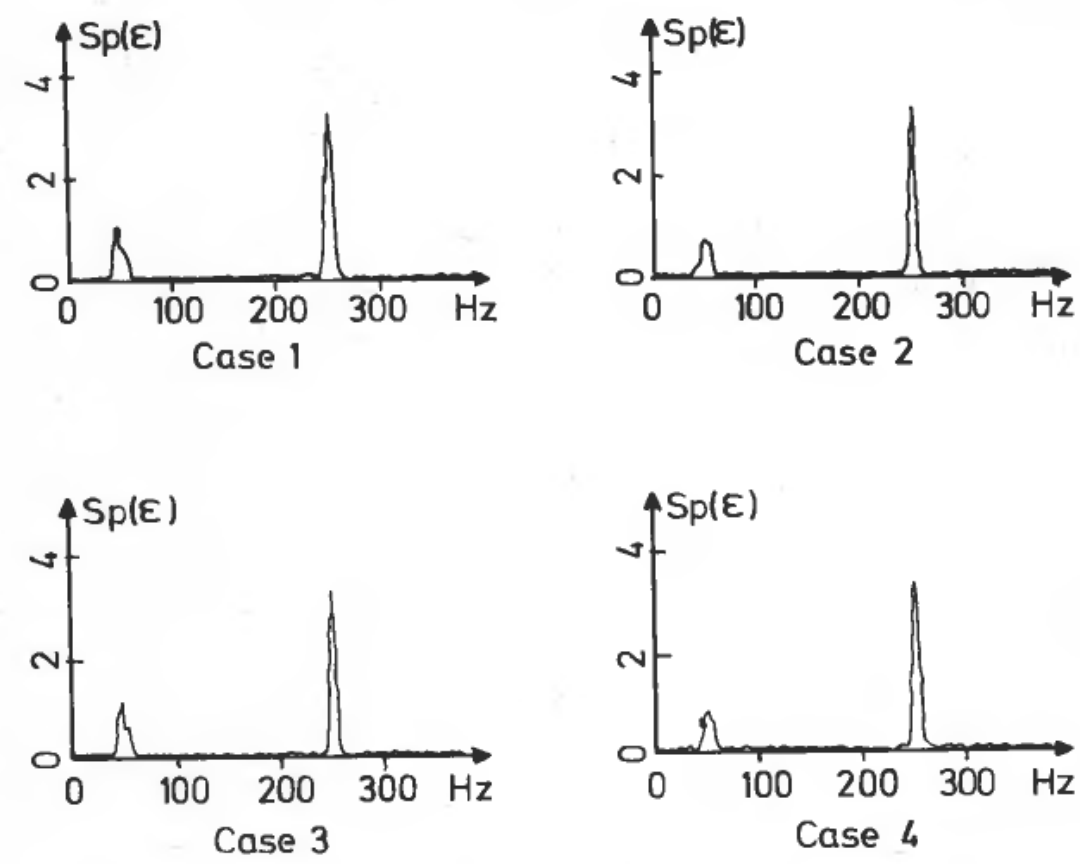

Figure 6. Power spectra of the innovations for all the cases in Table 2.

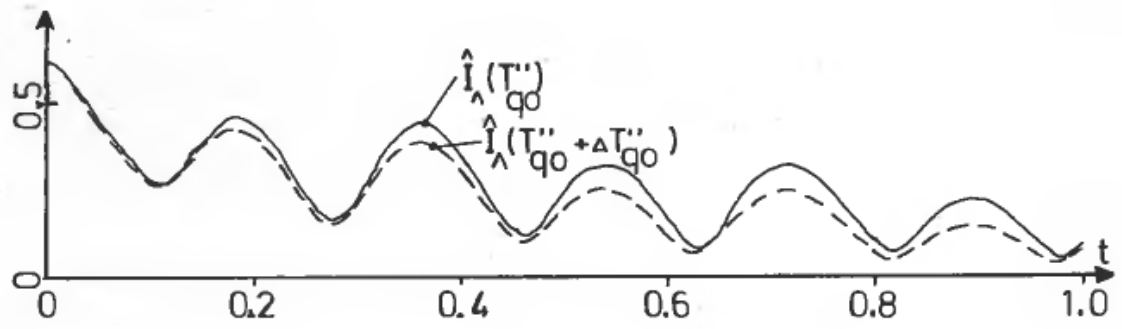

Figure 7. Sensitivity of the envelope of predicted measurement to a $+50 \%$ change in the time constant $T^{\prime \prime}{ }_{\text {q0. }}$ 


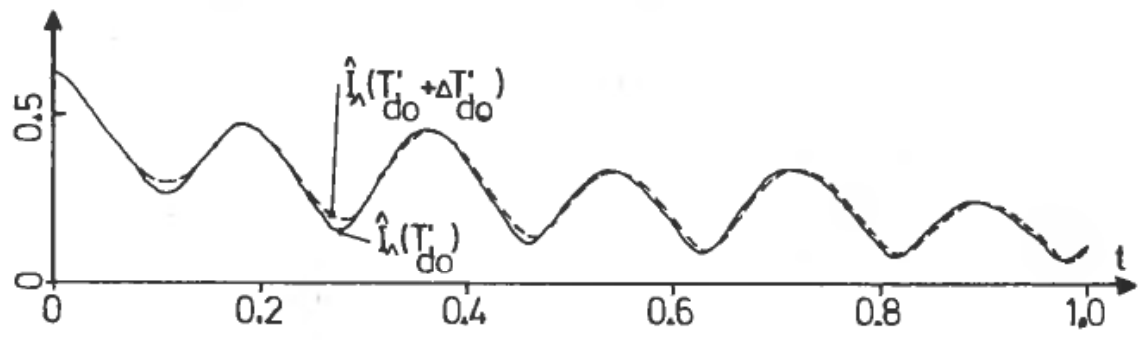

Figure 8. Sensitivity of the envelope of predicted measurement to a $+50 \%$ change in the time constant $\boldsymbol{T}_{\text {do. }}^{\prime}$

(no estimate attempted) and $\lambda_{\mathbf{s}}$. In order to determine the degree of identifiability both qualitatively and quantitatively, we used the sensitivity and curvature programs described in $\S$ A.3 of the Appendix. Figures 7 and 8 indicate that $T^{\prime \prime}{ }_{40}$ has a higher degree of identifiability than $T^{\prime}{ }_{\mathrm{do}}$. This is confirmed by the curvature calculations for the parameters $M, T^{\prime \prime}{ }_{\text {qo }}, T^{\prime}{ }_{\text {do }}$ and $T^{\prime \prime}{ }_{\text {do }}$. The criterion alters by the same value if $M$ is changed by $1 \%, T^{\prime \prime}{ }_{\text {qo }}$ by $12 \%, T^{\prime}{ }_{\text {do }}$ by $19 \%$ or $T^{\prime \prime}{ }_{\text {do }}$ by $77 \%$. In the case of $T^{\prime \prime}{ }_{\text {do, }}$, its degree of identifiability is so low that measurement noise is likely to affect the estimate of its value.

\section{Conclusion}

It has been shown how several parameters in the transient stability model of the synchronous machine can be estimated by means of the maximum likelihood method. (Measurements from a laboratory machine were used.) For the short circuit test the ML method is able to estimate the q-axis subtransient reactance $x^{\prime \prime}{ }_{q}$, which is almost impossible by the conventional graphical method. The synchronization and close test showed the importance of estimating the q-axis subtransient time constant $T^{\prime \prime}{ }_{\mathrm{q}}$ in order to get the correct damping. The estimation results showed that $T^{\prime \prime}{ }_{\text {qo }}$ could easily be estimated for our system. Even though the synchronization and close test is not directly applicable to large synchronous machines, because an infinite bus does not exist, the identifiability analysis and approach used should be of general interest. (Simulations performed by Hallingstad (1978) show that the closed-loop identification of $T^{\prime \prime}{ }_{\text {qo }}$ and $x^{\prime \prime}{ }_{\mathrm{q}}$ is possible and should be relatively easy to implement even for large synchronous machines.)

\section{ACKNOWLEDGMENT}

This research was done while the author held a scholarship at the Norwegian Institute of Technology, Division of Engineering Cybernetics, Trondheim, Norway.

\section{Appendix: Estimation program}

\section{A.1. Program structure}

This appendix presents a short description of the program used to estimate parameters and examine their identifiability. The method and the interactive computer program has been fully described in Hallingstad (1976). The main structure of the estimation program is shown in Fig. 9. The program consists of four levels. 


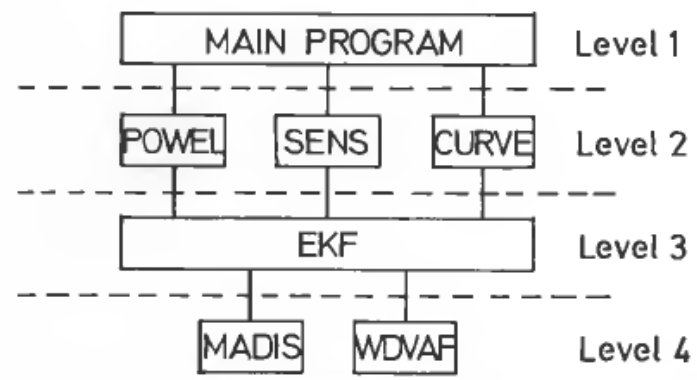

Figure 9. Main structure of the estimation program.

At level 1 initial values for the Kalman filter and the parameter vector are read into the memory along with the control variables for the routines at level 2 . These control variables define the parameters which are to be estimated, convergence limits for the minimization routine (POWELL) and parameter perturbations for sensitivity (SENS) and curvature (CURVE) calculations.

At level 2 we have the three main subroutines. POWELL calculates the parameter estimates by minimizing the maximum likelihood criterion, see eqn. (A 2). SENS performs sensitivity calculation by perturbing one parameter at a time. CURVE calculates the Hessian matrix, $H$, of the criterion at the parameter values $p$ :

$$
H(p)=\frac{\partial^{2} J(N: p)}{\partial p \partial p^{T}}
$$

These calculations are performed numerically.

At level 3 is the subroutine EKF (extended Kalman filter) which solves the extended Kalman filter equations and calculates the criterion.

$$
J(N: p)=\frac{1}{2} \sum_{i=1}^{N}\left[\epsilon_{1}^{\mathrm{T}} R_{i}^{-1} \epsilon_{i}+\ln \left(\operatorname{det} R_{1}\right)\right]
$$

where

$$
\begin{aligned}
N: & \text { number of samples } \\
\boldsymbol{p}: & \text { unknown parameter vector } \\
\boldsymbol{\epsilon}_{\mathrm{i}}=\boldsymbol{y}_{\mathrm{i}}-\overline{\boldsymbol{y}}_{\mathrm{i}}: & \text { innovation process } \\
R_{i}: & \text { covariance matrix for the innovation process }
\end{aligned}
$$

At level 4 the most important subroutines are MADIS and WDVAF. MADIS displays chosen time functions such as measurements $(y)$, predicted measurements $(\bar{y})$, innovations $(\boldsymbol{\epsilon})$ and estimates $(\hat{x})$, on the display. The same time functions may be written on file by means of WDVAF (Write Displayed VAriables on File) enabling the use of library programs on these files. For instance, hard copies may be made of the displayed variables on the Versatec plotter, in addition to plots of the autocorrelation and power spectra.

\section{A.2. Estimation algorithm}

There exist several methods for estimating parameters in dynamic processes. The maximum likelihood (ML) method leads to an algorithm with several advantages. In addition, two of the most frequently used least squares (LS) methods, i.e. the 
equation error method and the output error method, may be considered as special cases of the ML-method.

The maximum likelihood estimate, $\hat{p}$, of an unknown parameter vector, $p$, is defined as the parameter vector which maximize the joint probability function $(f(\cdot))$ of all the measurements, $Y_{N}($ Schweppe 1973),

$$
f\left(Y_{N}: \hat{p}\right)=\max _{p} f\left(Y_{N}: p\right)
$$

If we use Bayes formula and assume the innovation process, $\mathbf{\epsilon}_{\mathrm{i}}=\boldsymbol{y}_{\boldsymbol{i}}-\overline{\boldsymbol{y}}_{\boldsymbol{i}}$, at time $\boldsymbol{i}$ to be white, the maximum likelihood estimate can be determined as the parameter vector which minimize the criterion of eqn. (A 2). In order to calculate the MLestimate

(i) the criterion (eqn. (A 2)) must be calculated; and

(ii) the criterion (eqn. (A 2)) must be minimized.

The first problem can be solved using an ordinary Kalman filter for linear dynamic systems and an extended Kalman filter for non-linear systems.

There are many ways to minimize the criterion (eqn. (A 2)) for linear systems (see Gupta and Mehra 1974), but for non-linear systems the problem gets more involved. We have chosen Powell's method (Powell 1964) because this method does not need calculation of derivatives.

The main structure of the ML-estimator is shown in Fig. 10.

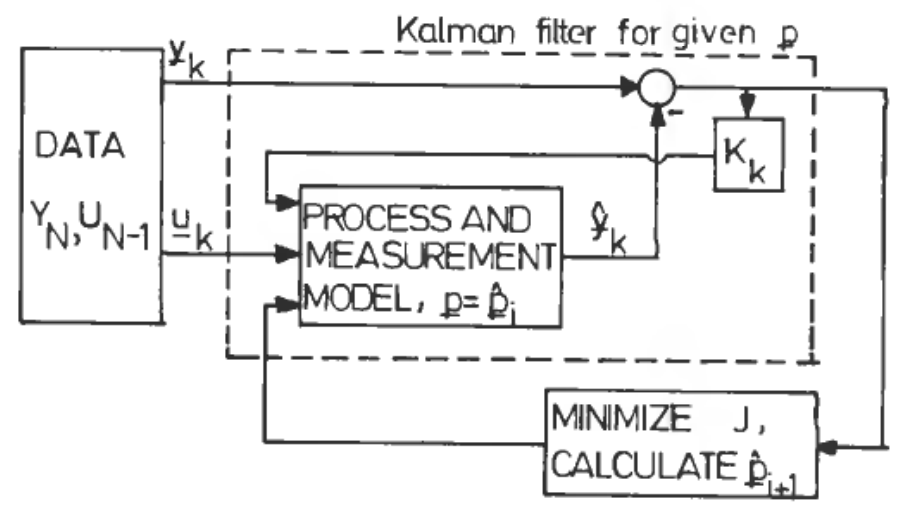

Figure 10. Structure of the ML-estimator.

\section{A.3. Identifiability tests}

The estimation program contains two subroutines which are used to check the identifiability of the parameters.

The subroutine SENS calculates numerically the sensitivity function

for the system given by

$$
s_{i}(t)=\frac{\partial y(t)}{\partial p_{i}}
$$

with measurements

$$
\dot{x}=f(x, p), \quad x(0)=x_{0}
$$

$$
y=g(x, p)
$$


In addition to the time function $s_{i}(t)$ we may display $y(t)$ when the parameter vector is equal to $p$ and when it is equal to $p+\Delta p$. By comparing the sensitivity functions $s_{i}$ and $s_{j}$ we may get a qualitative measure of whether $p_{i}$ or $p_{j}$ is more closely identifiable. In order to be identifiable the parameter $p_{t}$ must have a nonvanishing sensitivity function.

The subroutine CURVE performs a numerical calculation to determine the curvature of the criterion at parameter value $p$, i.e. the Hessian matrix

$$
H(p)=\frac{\partial^{2} J(N: p)}{\partial p \partial p^{T}}
$$

When $H(p)$ is a minimum $\partial \boldsymbol{J} / \partial \boldsymbol{p}=\boldsymbol{0}$, a quadratic approximation to the criterion increment is given by

$$
\Delta J \approx \frac{1}{2} \Delta p^{\mathrm{T}} H\left(p_{0}\right) \Delta p
$$

where $p_{0}$ indicates the parameter vector at the minimum. Since eqn. (A 8) has a quadratic form, $\Delta \boldsymbol{J}=$ constant defines an ellipsoid in the $n_{\mathrm{p}}$-dimensional parameter space. The directions and lengths of the half-axes are given by $m_{i}$ and $\lambda_{i}^{-1 / 2}$, where $m_{i}$ is the eigenvector belonging to eigenvalue $\lambda_{i}\left(i=1,2, \ldots, n_{\mathrm{p}}\right)$. Figure 11 shows a second-order example (assuming $H$ is positive definite).

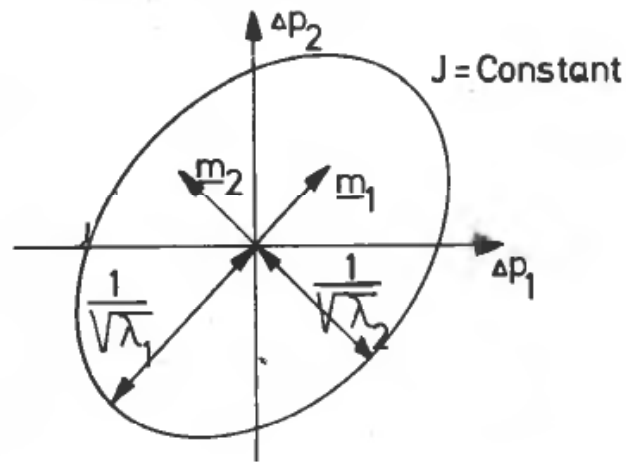

Figure 11. Interpretation of a positive definite Hessian matrix.

The surface of the ellipsoid has the characteristic that all parameter increments terminating on the surface give the same criterion increment. This means that if $\lambda_{i}>\lambda_{j}$ the parameter increments $\Delta \boldsymbol{p}_{\boldsymbol{i}}=c \cdot \boldsymbol{m}_{\boldsymbol{i}}$ are more identifiable than the increments $\Delta p_{k}=c \cdot m_{j}$ because $\Delta p_{i}$ gives a greater increment of the criterion than $\Delta p_{j} \cdot$

\section{REFERENCES}

ANDERSON, O. W. (1976). Generalized theory of rotating electrical machines. Norwegian Institute of Technology, Division of Electrical Machinery, Trondheim, Norway.

GuPtA, N., and MeHRA, R. (1974). Computational aspects of maximum likelihood estimation and reduction in sensitivity function calculations. I.E.E.E. Trans. autom. Control, 19, 774-783. 
HALlingSTAD, O. (1976). Maximum likelihood estimation of the parameters in non-linear state space models. SINTEF Report STF48 A76061, The Norwegian Institute of Technology, Division of Engineering Cybernetics, Trondheim, Norway (in Norwegian); (1978). Transient stability models: Parameter estimation and model reduction. Report 78-25-W, The Norwegian Institute of Technology, Division of Engineering Cybernetics, Trondheim, Norway.

Powell, M. J. D. (1964). An efficient method for finding the minimum of a function of several variables without calculating derivatives. Comput. J. 7, 155-162.

Schwepre, F. C. (1973). Uncertain Dynamic Systems (Prentice-Hall). 